\title{
GRBs in the comoving frame: interpreting the spectral-energy correlations
}

\author{
G. Ghirlanda ${ }^{1}$; G. Ghisellini ${ }^{1}$, L. Nava ${ }^{2}$, D. Burlon ${ }^{3}$, A. Celotti ${ }^{4}$, S. Covino ${ }^{1}$, A. \\ Melandri $^{1}$ \\ 1: INAF - Osservatorio Astronomico di Brera, Italy \\ 2: APC Université Paris Diderot, F-75205 Paris Cedex 13, France \\ 3: Sydney Institute for Astronomy, School of Physics, The University of Sydney, NSW 2006, \\ Australia \\ 4: SISSA - ISAS, Trieste, Italy
}

\begin{abstract}
We estimate the bulk Lorentz factor $\Gamma_{0}$ of 31 GRBs using the measured peak time of their afterglow light curves and considering a homogeneous circumburst medium or a wind density profile. The values of $\Gamma_{0}$ are distributed between few tens and several hundreds with average values $\sim 138$ and $\sim 66$ for the homogeneous and wind density profile, respectively. The isotropic energy and luminosity correlate in a similar way with $\Gamma_{0}$, i.e. $E_{\text {iso }} \propto \Gamma_{0}^{2}$ and $L_{\text {iso }} \propto \Gamma_{0}^{2}$, while the peak energy $E_{\mathrm{p}} \propto \Gamma_{0}$. These correlations are less scattered in the wind density profile than in the homogeneous case. We then study the energetics, luminosities and spectral properties of our bursts in their comoving frame. The distribution of $L_{\text {iso }}^{\prime}$ is very narrow with a dispersion of less than a decade in the wind case, clustering around $L_{\text {iso }}^{\prime} \sim 5 \times 10^{48} \mathrm{erg} \mathrm{s}^{-1}$. Peak photon energies cluster around $E_{\mathrm{p}}^{\prime} \sim$ $6 \mathrm{keV}$. The newly found correlations involving $\Gamma_{0}$ allow us to interpret the $E_{\mathrm{p}}-E_{\text {iso }}$ and $E_{\mathrm{p}}-L_{\text {iso }}$ correlations as due to the different $\Gamma_{0}$ factors and the collimation-corrected correlation, $E_{\mathrm{p}}-E_{\gamma}$ (obtained by correcting the isotropic quantities for the jet opening angle $\theta_{\mathrm{j}}$ ), can be explained if $\theta_{\mathrm{j}}^{2} \Gamma_{0}=$ constant. Assuming the $E_{\mathrm{p}}-E_{\gamma}$ correlation as valid, we find a typical value of $\theta_{\mathrm{j}} \Gamma_{0} \sim$ $6-20$, in agreement with the predictions of magnetically accelerated jet models.
\end{abstract}

Gamma-Ray Bursts 2012 Conference-GRB2012,

May 07-11, 2012

Munich, Germany

\footnotetext{
* Speaker.

${ }^{\dagger}$ E-mail: giancarlo.ghirlanda@brera.inaf.it
} 


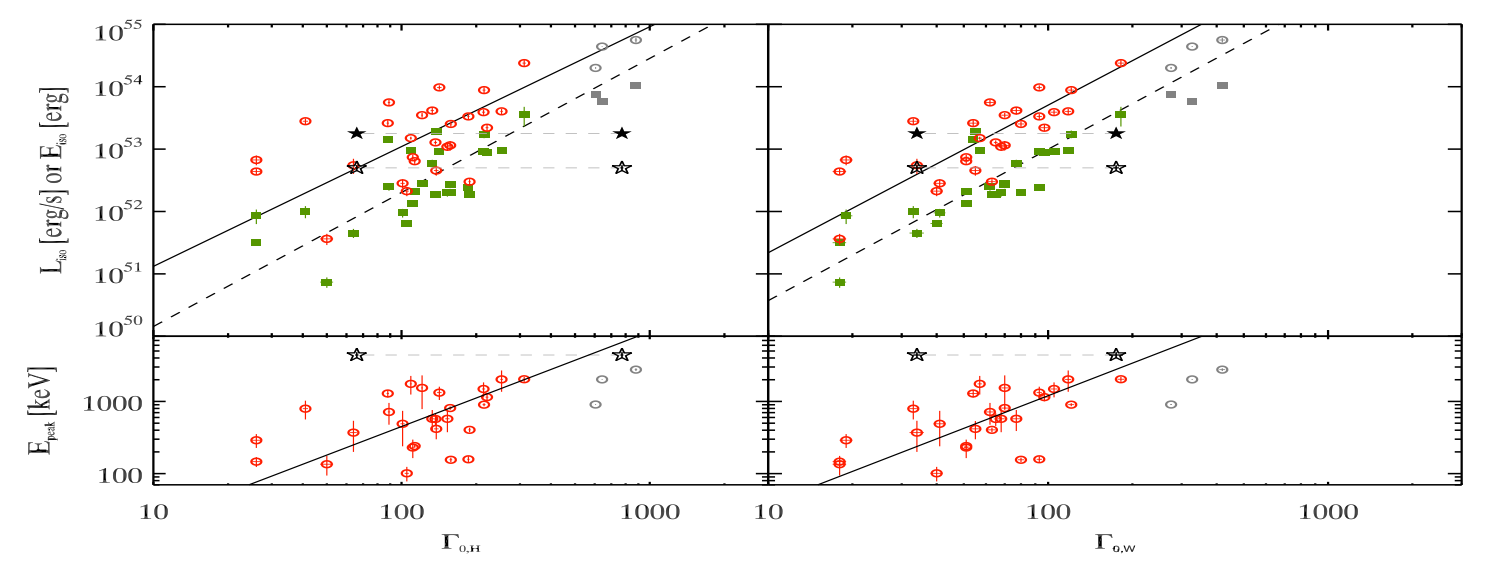

Figure 1: Top panels: Isotropic equivalent energy $E_{\text {iso }}$ (open circles) and luminosity $L_{\text {iso }}$ (filled squares) as a function of $\Gamma_{0}$, computed for the 30 long GRBs in our sample in the Homogeneous ISM case (left panel) and for a wind density ISM (right panel). The solid (dashed) line in both panels show the least square fit with a power law to the $E_{\mathrm{iso}}-\Gamma_{0}\left(L_{\mathrm{iso}}-\Gamma_{0}\right)$ correlation to the sample of 27 GRBs with peak in the optical light curve (open red circles and filled green squares). The three GRBs with peak in the GeV light curve are shown with the grey symbols but are not included in the fits shown here. The short GRB 090510 with both a peak in the $\mathrm{GeV}$ and a delayed peak in the optical is shown by star symbols connected by the dashed (gray) line. The larger value of $\Gamma_{0}$ for 090510 is that derived from the peak in the GeV light curve. Bottom panels: Peak energy $E_{\mathrm{p}}$ for the $\mathrm{H}$ case (left panel) and $\mathrm{W}$ case (right panel) as a function of $\Gamma_{0}$. The solid line is the best fit correlation.

\section{1. $E_{\mathrm{iso}}, L_{\mathrm{iso}}$ and $E_{\mathrm{p}}$ correlations with $\Gamma_{0}$}

Among the 132 (up to May 2011) GRBs with measured $E_{\text {peak }}$ and known redshift we searched for those events with a peak of the afterglow light curve $t_{\mathrm{p}, \mathrm{z}}: 27 \mathrm{GRBs}$ have a peak in their optical light curve and 4 have a peak in their GeV light curve as observed by Fermi/LAT [6]. For these 31 GRBs we estimated [5] the initial bulk Lorentz factor $\Gamma_{0}$ considering two possible scenarios: a uniform interstellar medium density profile $(n=$ const, $\mathrm{H})$ or a wind density profile $\left(n \propto r^{-2}, \mathrm{~W}\right)$.

We have derived the peak energy $E_{\text {peak }}^{\prime}$, the isotropic energy $E_{\text {iso }}^{\prime}$ and the isotropic peak luminosity $L_{\text {iso }}^{\prime}$ in the comoving frame. For the wind case the $\Gamma_{0}$-distribution has a typical value $\left\langle\Gamma_{0}\right\rangle \sim$ 66 , whereas in the $\mathrm{H}$ case $\left\langle\Gamma_{0}\right\rangle \sim 138$. The distribution of $E_{\text {peak }}^{\prime}$ is relatively narrow and centered around $\sim 6 \mathrm{keV}$ or $\sim 3 \mathrm{keV}$ for the $\mathrm{W}$ and $\mathrm{H}$ case. The distribution of $L_{\text {iso }}^{\prime}$ clusters, especially for the wind case, in a very narrow range (much less than a decade), around $5 \times 10^{48} \mathrm{erg} \mathrm{s}^{-1}$, while the distribution of $E_{\text {iso }}^{\prime}$ is broader and centered at $3 \times 10^{51} \mathrm{erg}$.

We found that the GRB rest frame energetics $E_{\text {iso }}$ and $L_{\text {iso }}$ correlate similarly (Fig. 1 - top panels) with $\Gamma_{0}$ (i.e. $\left.\propto \Gamma_{0}^{2.2}\right)$ and these correlations are less scattered $(\sigma=0.07)$ for the wind case (Fig. 1 - top right panel). There is also a linear correlation between $E_{\mathrm{p}}$ and $\Gamma_{0}$ (Fig. 1 - bottom panels).

2. Comoving frame correlations and interpretations of the $E_{\mathrm{p}}-E_{\mathrm{iso}}, E_{\mathrm{p}}-L_{\text {iso }}$ and $E_{\mathrm{p}}-E_{\gamma}$ correlations

In Fig. 2 we show the $E_{\mathrm{p}}-E_{\text {iso }}$ correlation (left panel) and the $E_{\mathrm{p}}-L_{\text {iso }}$ correlation (right 


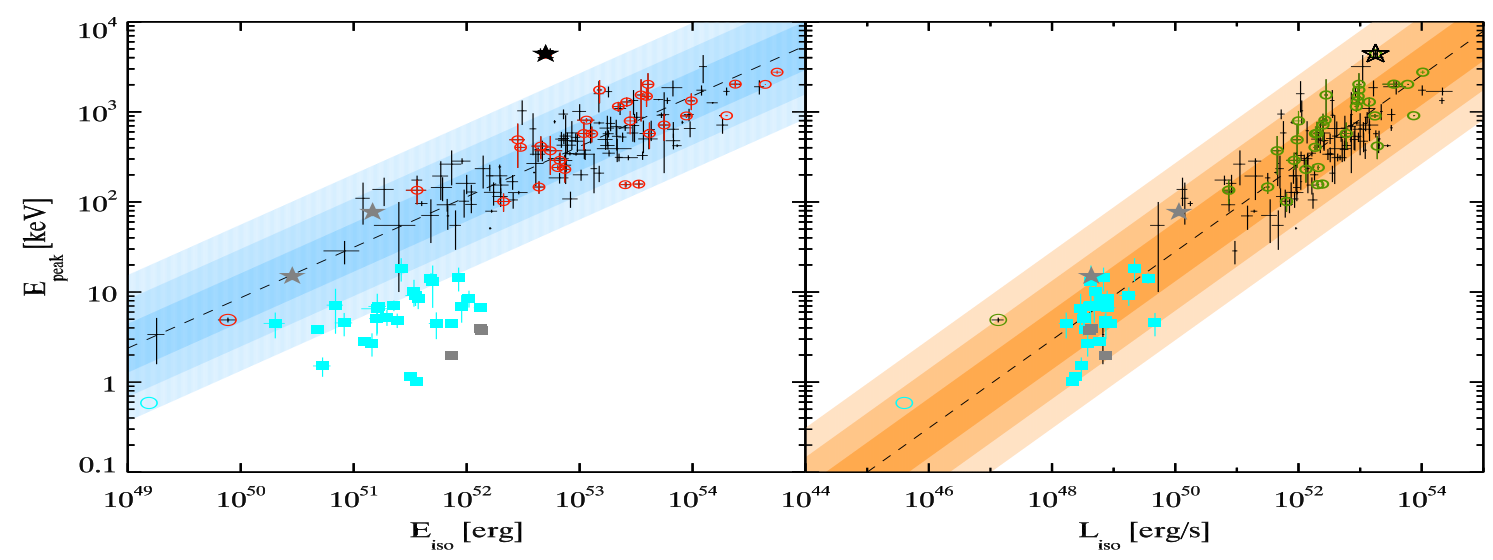

Figure 2: Wind density profile. Left: $E_{\mathrm{p}}-E_{\text {iso }}$ correlation in the rest frame (crosses and red circles) for 132 GRBs with $z$ and fitted $E_{\mathrm{p}}$ updated to May 2011. Right: $E_{\mathrm{p}}-L_{\text {iso }}$ correlation with $131 \mathrm{GRBs}$. In both panels the best fit correlation is shown by the dashed line and its $1,2,3 \sigma$ scatter is shown by the shaded region. The comoving frame $E_{\mathrm{p}}^{\prime}$ and $E_{\text {iso }}^{\prime}$ (left) and $E_{\mathrm{p}}^{\prime}$ and $L_{\text {iso }}^{\prime}$ (right) of $30 \mathrm{GRBs}$ (red open circles [left panel] and green open circles [right panel]) in our sample with an estimate of the $\Gamma_{0}$ factor are shown with the filled cyan square symbols ( 27 events with $t_{\mathrm{p}, \mathrm{z}}$ in the optical light curve) or grey filled square (the three long GRBs with a peak in the GeV light curve). The short GRB 090510 is also shown with a star symbol and the low luminosity GRB 060218 (with $\Gamma_{0} \sim 5$ [Ghisellini et al. 2006]) is shown with an open circle.

panel) where we correct the rest frame $E_{\mathrm{p}}$ and $E_{\text {iso }}$ or $L_{\text {iso }}$ for $\Gamma_{0}$. We find that there is a considerable clustering of the bursts with measured $\Gamma_{0}$ in the $E_{\mathrm{p}}-L_{\text {iso }}$ plane. The second column of the Tab. 1 reports some immediate implications of our results (see [4] for a complete discussion).

Since $E_{\text {peak }}^{\prime} \propto E_{\text {peak }} \Gamma_{0}$ is contained in a narrow range, all bursts emit their radiation at a characteristic frequency in their comoving frame, irrespective of their bulk Lorentz factor. This finding is supported by recent results on photospheric GRB emission [7].

If we assume that $E_{\text {peak }} \propto \Gamma_{0}$ (bottom panels of Fig. 1) together with the quadratic dependence on $\Gamma_{0}$ of $E_{\text {iso }}$ and $L_{\text {iso }}$ (top panels of Fig. 1) we find the "Amati" [1] and the "Yonetoku" [11] relations. They are a sequence of $\Gamma_{0}$-factors.

If all bursts had the same jet opening angle, then $L_{\gamma}^{\prime}=\theta_{\mathrm{j}}^{2} L_{\mathrm{iso}}^{\prime}$ and the (logarithmic) width of the $L_{\text {iso }}^{\prime}$ distribution would be the same of the (more fundamental) $L_{\gamma}^{\prime}$ distribution. On the other hand, we have some hints that very energetic and luminous GRBs tend to have narrower opening angles (e.g. [2]). It is this property that makes the collimation corrected $E_{\gamma}$ and $L_{\gamma}$ quantities to correlate with $E_{\text {peak }}$ in a different way (i.e. different slope) than in the Amati and Yonetoku relation.

We are then led to propose the following ansatz: the opening angle of the jet inversely correlates with the bulk Lorentz factor $\theta_{\mathrm{j}} \propto \Gamma_{0}{ }^{-a}$. There are too few GRBs in our sample with measured $\theta_{\mathrm{j}}$ and $\Gamma_{0}$ to find a reasonable value for the exponent $a$, but it is nevertheless instructive to explore the case $a=1 / 2$, leading to $\theta_{\mathrm{j}}^{2} \Gamma_{0}=$ constant. If we assume this relation we find, for the collimation corrected $E_{\gamma}=\theta_{\mathrm{j}}^{2} E_{\text {iso }} \propto \Gamma_{0} \propto E_{\text {peak }}$ This is the linear $E_{\mathrm{p}}-E_{\gamma}$ correlation in the wind case [9].

In our sample, only for 4 bursts we can estimate the jet opening angle from the measure of the jet break time of the optical light curve. Their small number does not make possible to directly test the existence of a relation between $\Gamma_{0}$ and $\theta_{\mathrm{j}}$. We have recently preformed Monte Carlo simulations [5] (see also Ghisellini et al. this conference) where we tested if there exists a relation between $\Gamma_{0}$ and $\theta_{\text {jet }}$ or not. We found [5] that the distributions of $\Gamma_{0}$ and $\theta_{\text {jet }}$ should have characteristic 


\begin{tabular}{lll}
\hline \hline Our results & Implications & If $\theta_{\mathrm{j}}^{2} \Gamma \sim$ const \\
\hline$E_{\text {peak }}^{\prime} \sim$ const & $E_{\text {peak }} \propto \Gamma$ & \\
$E_{\text {iso }} \propto \Gamma^{2}$ & $E_{\text {iso }} \propto E_{\text {peak }}^{2}$ & $E_{\gamma}=\theta_{\mathrm{j}}^{2} E_{\text {iso }} \propto \Gamma \propto E_{\text {peak }}$ \\
$L_{\text {iso }} \propto \Gamma^{2}$ & $L_{\text {iso }} \propto E_{\text {peak }}^{2}$ & $L_{\gamma}=\theta_{\mathrm{j}}^{2} L_{\text {iso }} \propto \Gamma \propto E_{\text {peak }}$ \\
$T_{90}$ not $f(\Gamma)$ & $T_{90}^{\prime} \propto \Gamma$ & $E_{\gamma}^{\prime} \sim$ const \\
$L_{\text {iso }}^{\prime} \sim$ const & $E_{\text {iso }}^{\prime} / L_{\text {iso }}^{\prime} \propto T_{90}^{\prime} \propto \Gamma$ & $L_{\gamma}^{\prime} \sim E_{\gamma}^{\prime} / T_{90}^{\prime} \sim 1 / \Gamma$ \\
\hline \hline
\end{tabular}

Table 1: Schematic summary of our results and their implications for the case of a wind density profile.

values and there should be a relation similar to the ansatz that we made above between these two parameters in order to reproduce samples of GRBs observed by different satellites. In the case of an homogeneous density profile the typical $\theta_{\mathrm{j}} \sim 0.1$ radiants [3] while in the case of a wind density profile $\theta_{\mathrm{j}} \sim 0.07$ radiants. Combining these values with the average values of $\Gamma_{0}$ estimated in our work we find $\theta_{\mathrm{j}} \Gamma_{0} \sim 14(5)$ for the $\mathrm{H}(\mathrm{W})$ case. These values are in good agreement with the results of recent simulations of (i) a magnetized jet confined by the stellar material that freely expands when it breaks out the star [8] or (ii) a magnetized unconfined split-monopole jet [10].

\section{References}

[1] L. Amati, F. Frontera, M. Tavani et al., Intrinsic spectra and energetics of BeppoSAX Gamma-Ray Bursts with known redshifts, A\&A 390) (2002) 81

[2] C. Firmani, G. Ghisellini, G. Ghirlanda, et al., A new method optimized to use gamma-ray bursts as cosmic rulers, MNRAS 360 (2005) L1

[3] G. Ghirlanda, G. Ghisellini \& D. Lazzati, The Collimation-corrected Gamma-Ray Burst Energies Correlate with the Peak Energy of Their $v F_{v}$ Spectrum, ApJ 616 (2004) 331

[4] G. Ghirlanda, L., Nava, L., G. Ghisellini et al., Gamma-ray bursts in the comoving frame, MNRAS 420 (2012) 483

[5] G. Ghirlanda, G. Ghisellini G., R. Salvaterra et al., The faster the narrower: characteristic bulk velocities and jet opening angles of Gamma Ray Bursts, subm to MNRAS (2012)

[6] G. Ghisellini , G. Ghirlanda , L. Nava , A. Celotti A., GeV emission from gamma-ray bursts: $a$ radiative fireball?, MNRAS $\mathbf{4 0 3}$ (2010) 926

[7] D. Giannios, The peak energy of dissipative gamma-ray burst photospheres, MNRAS 422 (2012) 3092

[8] Komissarov S. S., Vlahakis N., Koenigl A., Rarefaction acceleration of ultrarelativistic magnetized jets in gamma-ray burst sources, MNRAS 407 (2010) 17

[9] L. Nava , G. Ghisellini , G. Ghirlanda , et al., On the interpretation of spectral-energy correlations in long gamma-ray bursts, $A \& A 450$ (2006) 471

[10] Tchekhovskoy A., McKinney J. C., Narayan R., Efficiency of Magnetic to Kinetic Energy Conversion in a Monopole Magnetosphere, ApJ 699 (2009) 1789

[11] D. Yonetoku, T. Murakami, T. Nakamura, et al., Gamma-Ray Burst Formation Rate Inferred from the Spectral Peak Energy-Peak Luminosity Relation, ApJ 609 (2004) 935 